\title{
TECNOLOGÍAS DE INFORMACIÓN PARA ACERCAR AL CIUDADANO A LOS SERVICIOS DE JUSTICIA EN COLOMBIA: EL CASO DEL MAPA DE OFERTA DE JUSTICIA
}

\author{
INFORMATION TECHNOLOGIES TO BRING CLOSER CITIZENS TO JUSTICE SERVICES IN \\ COLOMBIA: THE CASE OF THE MAP OF OFFER OF JUSTICE SERVICES
}

\author{
Ernesto Amaru Galvis Lista \\ Ingeniero de Sistemas, MSc, Ph.D. (C), Profesor Asistente, Facultad de Ingeniería. \\ Universidad del Magdalena, Santa Marta, Colombia \\ egalvis@unimagdalena.edu.co \\ Mayda Patricia González Zabala \\ Ingeniera de Sistemas, MSc, Ph.D. (C), Profesora Asistente, Facultad de Ingeniería. \\ Universidad del Magdalena, Santa Marta, Colombia \\ mpgonzalez@unimagdalena.edu.co \\ Pablo Hernán Vera Salazar \\ Ingeniero Civil, MSc, Ph.D. (C), Profesor Auxiliar, Facultad de Ingeniería. \\ Universidad del Magdalena, Santa Marta, Colombia \\ pvera@unimagdalena.edu.co
}

Fecha de recepción: 12 de septiembre de 2011

Fecha de aprobación: 19 de diciembre de 2011

\section{RESUMEN}

El propósito de este artículo es presentar los resultados de un proyecto de desarrollo tecnológico que consistió en construir una plataforma web para administrar y divulgar de la información sobre la oferta de servicios de justicia en Colombia, denominada Mapa de Oferta de Justicia. Para lograr los resultados, se utilizó un enfoque ágil en el desarrollo del software, específicamente, el método denominado programación extrema (extreme programming - XP. Los principales resultados fueron: la construcción de tres productos software: (1) tesauro de oferta de justicia, (2) directorio de instituciones, y (3) plataforma de reportes e indicadores sobre oferta de justicia; y la recopilación de la información de contacto y caracterización de cada institución que ofrece servicios de justicia en Colombia. Por último, este trabajo muestra la forma como la tecnología de información puede apoyar áreas tan importantes como la justicia en Colombia, lo cual está en consonancia con lo propuesto en el Plan Nacional de Tecnologías de la Información y las Comunicaciones (Colombia Plan TIC).

Palabras clave: tecnologías de información, TIC, oferta de servicios de justicia

\begin{abstract}
The purpose of this paper is to present the results of a technological development project which consist in building a web platform to support management and divulgation of
\end{abstract}


information about the offer of justice services in Colombia, denominated Map of Justice Offer. To obtain the results, we used an agile approach to software development, specifically, a method named extreme programming - XP. The main results were building three software products: (1) Thesaurus of Justice Offer, (2) Directory of Institutions, and (3) a platform of indicators and reports about justice offer: and gathering contact information and a characterization of each institution that offers justice senvices in Colombia. Last, this work shows a way in that information technology could bring support to very important public sectors like justice in Colombia, which is aligned to the National Plan of Information and Communication Technologies - (Colombia Plan TIC).

Keywords: information technology, ICT, offer of justice services.

\section{INTRODUCCIÓN}

En Colombia, gran parte de la población no conoce los servicios de justicia que brinda el Estado. Tampoco conoce el tipo de instituciones jurídicas a las cuales pueden acudir en el caso de necesitar algún servicio u orientación. Además, el Ministerio del Interior y Justicia (MIJ), hasta principios de 2008, tenía dificultades para contar con la información detallada y actualizada de las instituciones que ofrecen servicios de justicia, su ubicación exacta, su información de contacto, en cada municipio del territorio nacional, Vicerrectoría de Extensión [1].

La brecha en la información sobre la presencia institucional, los servicios que cada institución ofrece a la población y la forma de acceder a dichos servicios; además de limitar el acceso, constituye una barrera que dificulta hacer estudios para vislumbrar el estado del sector, su comportamiento, la presencia institucional en cada región y su relación con el número de habitantes. También dificulta la definición de indicadores válidos que permitan identificar conflictos de jurisdicción, suficiencia o insuficiencia de la oferta de justicia por tipo de institución, por sectores o áreas de la justicia, entre otros, Vicerrectoría de Extensión [1].

Este problema se debe a la cantidad y diversidad de actores involucrados en ofrecer servicios de justicia, la diversidad de ámbitos territoriales que tiene el País, la escasa infraestructura para el manejo de la información que tienen algunos municipios, el manejo local que se le da a la información, la ausencia de mecanismos que permitan la actualización, consolidación y divulgación de la información, y la falta de estrategias para establecer canales efectivos de comunicación entre los diferentes actores y procedimientos sencillos para actualizar la información, Vicerrectoría de Extensión [1].

La principal consecuencia del problema del manejo de esta información es que se genera una visión borrosa de la oferta de justicia. Lo anterior hace que la población y el mismo sector no conozcan a fondo cuáles son los servicios que se ofrecen, qué funciones cumple cada institución y cuál es la disponibilidad de senvicios que se ofrecen en el País. Dificulta y hace 
compleja la definición de estrategias y la toma de decisiones orientadas a fortalecer y transformar el sector, Vicerrectoría de Extensión [2].

En este sentido, la Vicerrectoría de Extensión y el Grupo de Investigación en Organizaciones, Sistemas y Computación (GIDOSC) de la Universidad del Magdalena, ejecutaron un convenio interadministrativo firmado con el MIJ, cuyo objetivo fue recopilar la información sobre la oferta de justicia en Colombia y construir herramientas informáticas para apoyar su administración. Como el problema no era sólo jurídico ni técnico, el equipo de trabajo encargado de la ejecución del proyecto fue un equipo interdisciplinario conformado por profesionales y estudiantes de las disciplinas del Derecho y la Ingeniería de Sistemas. Con estas perspectivas complementarias, el equipo de trabajo logró tratar la problemática, cumpliendo con los objetivos establecidos en el convenio interadministrativo, y presentando a la sociedad una solución informática que, haciendo uso de las tecnologías de Internet, pone a disposición del ciudadano la información necesaria para lograr mayor claridad sobre la oferta de justicia, de modo que estos servicios puedan ser usados de forma efectiva.

Este artículo reporta a la comunidad académica, los resultados del proyecto Mapa de Oferta de Justicia en Colombia. Por lo tanto, se inicia con la presentación de la situación de interés, en donde se describen los elementos de indagación definidos por el equipo jurídico del proyecto, como las variables de interés sobre la oferta de justicia, los grupos de instituciones incluidas en el estudio, y la definición del alcance de la solución informática desarrollada. Luego, se presenta la metodología de desarrollo de software utilizada para guiar la construcción de las aplicaciones que conforman la solución informática. Seguidamente, se presentan los resultados obtenidos, en donde se describen las características de las tres aplicaciones software que conformaron la solución tecnológica implementada. Por último, se presentan las conclusiones de este trabajo y las referencias utilizadas en la elaboración del artículo.

\section{SÍNTESIS DE LA SITUACIÓN DE INTERÉS}

La administración de la información sobre la oferta de justicia en Colombia es una actividad que involucra diversos actores en varios ámbitos territoriales. Cada uno de los actores posee información parcial sobre las características de los servicios ofrecidos y la manera de acceder a ellos. Por ejemplo: cada universidad que ofrece el programa de Derecho, debe tener un consultorio jurídico y un centro de conciliación, y aunque los datos de funcionamiento deben reportarse a varias instituciones del ámbito local, regional y nacional, características particulares del funcionamiento y los servicios ofrecidos, solamente están disponibles para los usuarios y la población geográficamente cercana a la institución.

Esta desintegración de información causa efectos negativos como la ausencia de datos exactos y consolidados de la oferta de justicia en cada municipio del País, la inexistencia de estudios en donde se relacionen los datos de oferta de justicia utilizando varias dimensiones de análisis, la ausencia de estudios de campo que permitan entender integralmente la división de 
competencias por jurisdicción territorial y funcional, la inexistencia de indicadores validados que permitan identificar conflictos de jurisdicción, ausencia, sobreoferta o carencia de oferta de justicia institucional, y la ausencia de herramientas que relacionen la información demográfica (datos del número de habitantes), respecto de la oferta de justicia.

Conscientes de esta problemática, el MIJ, en el marco del programa de Fortalecimiento de la Justicia financiado por la Unión Europea, auspició la ejecución de un proyecto orientado a la búsqueda de la integración informacional. El proyecto titulado Proyecto Mapa de Justicia y Diplomado para gestores Municipales del Programa Nacional de acceso a la Justicia"ejecutado por la Vicerrectoría de Extensión de la Universidad de Magdalena [2], tuvo como propósito diseñar y brindar un programa de formación (diplomado) para gestores municipales, y buscar y recopilar, en el territorio nacional, la información referente a: ubicación, teléfonos de contacto, funcionario principal, funciones y competencias de la institución, servicios que ofrece, costos y carácter del servicio, áreas del derecho y cuantías.

Según el convenio interadministrativo que dio vida al proyecto, esta información se debería presentar de acuerdo con las siguientes categorías de instituciones: (1) instituciones de carácter judicial, (2) instituciones del ejecutivo, (3) organismos de control y (4) colaboradores de la justicia. Por otra parte, el proyecto tuvo como objetivo construir un tesauro de situaciones de consulta jurídica frecuente. En síntesis, en la ejecución de este proyecto se tenía estipulado que los resultados y productos entregables serían: la información actualizada de las instituciones oferentes de justicia en el País, el tesauro (términos relacionados) y un análisis de la información recopilada, Vicerrectoría de Extensión [2].

Tabla 1. Variables utilizadas en la caracterización de la oferta de justicia

\begin{tabular}{|c|l|}
\hline Tipos de Variables & \multicolumn{1}{c|}{ Descripción } \\
\hline Presencia institucional & Que exista una infraestructura física que permita prestar los servicios de justicia. \\
\hline Cobertura & $\begin{array}{l}\text { El porcentaje de unidades de población atendida en un periodo definido (un } \\
\text { año, por ejemplo) respecto de aquella potencialmente usuaria. (Con base en los } \\
\text { datos demográficos ya existentes) }\end{array}$ \\
\hline Calidad de la oferta & $\begin{array}{l}\text { Determinar hasta qué nivel la actividad del operador coadyuva eficientemente a } \\
\text { la resolución de conflictos, y si la manera como se presta sus servicios es } \\
\text { eficiente. (oportunidad, cercanía, personal etc.). }\end{array}$ \\
\hline Pertinencia & $\begin{array}{l}\text { Comparación entre los datos de calidad de oferta con respecto de la } \\
\text { conflictividad del territorio. }\end{array}$ \\
\hline
\end{tabular}

Fuente: Elaboración propia con base en Vera et al, 2008

En este sentido, el proyecto Mapa de Justicia se orientó a tratar de responder a estas situaciones problema, para lo cual se plantearon las siguientes preguntas de investigación: (1) ¿Cuáles son los servicios de justicia que actualmente ofrece el Estado? (2) ¿Cuáles son los servicios de justicia que actualmente ofrecen los operadores privados?, (3) ¿Dónde se encuentran los servicios de justicia que actualmente ofrece el Estado? (4) ¿Dónde se 
presentan los principales problemas de oferta de justicia por operadores públicos y privados? (5) ¿Dónde se presenta sobreoferta de instituciones e instancias?, (6) ¿Dónde se presenta carencia de oferta de instituciones e instancias? Y (7) ¿Cuáles son los datos y procedimientos que debe conocer el usuario a fin de acceder eficientemente a las instancias de oferta de justicia? De igual manera, se definieron las variables de trabajo, las cuales se presentan en la Tabla 1, Vera et al [3].

En relación con los sujetos de análisis, el proyecto consideró como universo de estudio las instituciones estatales y no estatales. Se identificaron 47 tipos de instituciones que funcionan como operadores de justicia, en rango nacional y local. Para su estudio las instituciones se agruparon como se presenta en la Tabla 2, Vera et al [3]. Además, se definió como unidad de análisis del ámbito geográfico el Municipio y sólo nombra los corregimientos cuando la oferta de justicia de un Municipio determina una jurisdicción espacial que requiera su inclusión, Vera et al [3]. Por lo tanto, se tomó la codificación de municipios realizada por el Departamento Administrativo Nacional de Estadística (DANE), DANE [4].

Tabla 2. Grupos de instituciones que ofrecen servicios de justicia

\begin{tabular}{|c|c|c|c|}
\hline Grupo & Nombre & Descripción & Ejemplo \\
\hline 1 & $\begin{array}{l}\text { Instituciones del } \\
\text { Poder Judicial }\end{array}$ & $\begin{array}{l}\text { Instituciones cuyo rango de acción, jurisdicción y } \\
\text { competencias están determinados en el estatuto } \\
\text { orgánico de la rama judicial y cuyo funcionamiento se } \\
\text { relaciona con la Ley } 270 \text { Estatutaria de la } \\
\text { Administración de Justicia. }\end{array}$ & $\begin{array}{l}\text { Consejo superior de la } \\
\text { Judicatura } \\
\text { Juzgados de circuito } \\
\text { Juzgados promiscuos }\end{array}$ \\
\hline 2 & $\begin{array}{l}\text { Instituciones del } \\
\text { Poder Ejecutivo }\end{array}$ & $\begin{array}{l}\text { Instituciones dentro de la órbita administrativa estatal, } \\
\text { ministerios o adscritos a él, del ámbito central o } \\
\text { descentralizado (institutos, centros, oficinas) dentro de } \\
\text { cuyas funciones se ha determinado, servir de operador } \\
\text { de justicia. }\end{array}$ & $\begin{array}{l}\text { Alcaldías, } \\
\text { Comisarías de familia } \\
\text { Instituto Nacional } \\
\text { Penitenciario }\end{array}$ \\
\hline 3 & $\begin{array}{l}\text { Organismos de } \\
\text { control }\end{array}$ & $\begin{array}{l}\text { Instituciones que tienen la función de controlar el } \\
\text { cumplimiento de las normas y las leyes mediante la } \\
\text { aplicación de principios técnicos y científicos. Los } \\
\text { organismos de control se establecen en el artículo } 117 \\
\text { de la Constitución Política de } 1991 \text {. }\end{array}$ & $\begin{array}{l}\text { Procuraduría General de } \\
\text { la Nación } \\
\text { Contraloría General de la } \\
\text { República } \\
\text { Defensoría del pueblo }\end{array}$ \\
\hline 4 & $\begin{array}{c}\text { Colaboradores } \\
\text { de la Justicia }\end{array}$ & $\begin{array}{l}\text { Instituciones que ofrecen servicios de justicia que logran } \\
\text { constituirse en instancias de resolución de conflictos } \\
\text { cuya eficacia es validada socialmente. Son en su gran } \\
\text { mayoría, de índole privado. }\end{array}$ & $\begin{array}{l}\text { Consultorios jurídicos } \\
\text { Centros de conciliación } \\
\text { Asociaciones de usuarios }\end{array}$ \\
\hline
\end{tabular}

Fuente: Elaboración propia con base en Vera et al, 2008.

La especificación del proyecto permitió apreciar que el volumen y detalle de la información por recopilar era alto. En este sentido, el Grupo GIDOSC y la Vicerrectoría de Extensión de la Universidad del Magdalena, conscientes de que se podría hacer un aporte mayor desde la academia, diseñaron e implementaron una solución informática que diera cuenta no sólo de los resultados y productos convenidos, sino que permitiera dinamizar el procesos de administración de la información. Por esta razón, se planteó una solución TI consistente en una plataforma web para acercar a los ciudadanos a los servicios del Estado. 
Este tipo de soluciones tecnológicas se enmarcan en una de las áreas de aplicación de la informática que se ha denominado Informática jurídica y que se enfoca principalmente en brindar el soporte informático necesario para apoyar la gestión de información jurídica, según Pérez-Luño et al [5], en tres aspectos: (1) las fuentes de información jurídica, también llamada informática jurídica documental; (2) la gestión de las tareas y actividades procedimentales, también llamada informática jurídica de gestión; y (3) el soporte en la toma de decisiones jurídicas, también llamada informática jurídica decisional. En particular, el objetivo de este proyecto fue brindar una solución tecnológica dentro del ámbito de la informática jurídica documental, pues se centró en la gestión de fuentes de información jurídica sobre la oferta de justicia en Colombia, y la forma en que los ciudadanos pueden acceder a ella.

Ahora bien, desde la perspectiva general de los Sistemas de Información - (SI) como disciplina, este proyecto se ubica en una de las áreas de mayor desarrollo en los últimos años: los SI en la Web. El componente computacional de estos sistemas de información está basado en aplicaciones software que se despliegan en infraestructuras conectadas a Internet o en una intranet corporativa, y que son accedidas por los usuarios utilizando un software para navegar en Internet. Este tipo de aplicaciones ha evolucionado en muy poco tiempo y ha demostrado ser una alternativa segura y versátil para la mayoría de las organizaciones.

Con este tipo de SI en la web, se ha generado la posibilidad de acercar al ciudadano a los diferentes servicios que ofrece el Estado y los entes privados. En ese sentido, se han promovido diferentes iniciativas tales como Gobierno en línea, que cuenta con un portal web denominado Portal del Estado colombiano ', en donde se suministra información general sobre las entidades del Estado, principalmente del ámbito nacional, y sobre los trámites asociados a cada una de las entidades. En particular, para el ámbito jurídico que es el centro de interés de este proyecto, el portal del Estado colombiano ofrece información muy general sobre los trámites por realizar en las instituciones públicas que ofrecen servicios de justicia en Colombia, enfocándose principalmente en las entidades de rango nacional. La mayor debilidad de este portal en cuanto a la información sobre la oferta de justicia es que no incluye información sobre entidades de naturaleza privada que también ofrecen servicios a los ciudadanos como los consultorios jurídicos, centros de conciliación, y otros tipos de instituciones clasificadas como colaboradores de la justicia. Además, no incluye una guía terminológica que siva de ayuda a la gran mayoría de ciudadanos que no tienen conocimientos jurídicos básicos y se les limita las posibilidades de consulta, al no tener claridad sobre los términos por utilizar.

Otro antecedente para destacar es el Sistema de Información de la Conciliación, que fue desarrollado y administrado por el MIJ, y actualmente se encuentra a cargo del Ministerio de Justicia y del Derecho. Este sistema se enfoca en la recopilación de información sobre la conciliación en Colombia. En particular, permite el registro de datos estadísticos sobre los

\footnotetext{
${ }^{1}$ En www.gobiernoenlinea.gov.co
} 
procesos de conciliación efectuados en las diferentes instituciones públicas y privadas que están habilitadas por la ley para ofrecer los servicios de conciliación. En este sentido, el portal, que da soporte informático al sistema de información de la conciliación ${ }^{2}$, ofrece la posibilidad de actualización y consulta de datos de las entidades que ofrecen este tipo de servicios de justicia. Además, ofrece la posibilidad de hacer consultas utilizando mapas, de modo que el ciudadano pueda acceder a la información, de acuerdo con su ubicación geográfica. Sin embargo, la principal debilidad de este sistema de información es que se centra en una parte de la oferta de justicia y deja por fuera otros servicios e instituciones que también sirven a los ciudadanos. No obstante, es importante destacar que para el desarrollo del Mapa de Oferta de Justicia en Colombia, es el referente institucional de mayor preponderancia.

En el ámbito internacional, se tomaron inicialmente tres referentes importantes para el desarrollo del proyecto como son el portal del Ministerio de Justicia de España ${ }^{3}$, el portal del Ministerio de Justicia de Chile ${ }^{4}$ y el portal del Ministerio de Justicia de Argentina ${ }^{5}$. En estos portales, los ciudadanos pueden acceder a un directorio de instituciones que ofrecen servicios de justicia y realizar búsquedas por diferentes criterios. Sin embargo, la principal debilidad que se detectó es que la información registrada de cada institución es muy general y no se ofrece una guía que facilite a las personas con pocos conocimientos sobre la terminología jurídica, poder ubicar de forma efectiva la institución a la cual acudir para resolver sus problemas jurídicos.

Por otra parte, se destaca un proyecto similar a la presente propuesta, adelantado en la

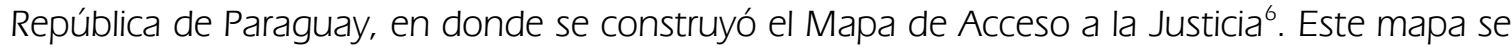
enfocó en recopilar información de las diversas instituciones oferentes de justicia ubicadas en Asunción, capital de la república de Paraguay. Allí se puede ubicar la dirección y algunos datos básicos de contacto para poder acceder a los servicios de justicia. Además, la información recopilada fue publicada en un documento titulado Mapa de Oferta de Justicia. La principal ventaja que se detectó en este servicio es la utilización de tecnología de mapas, en específico Google Maps, para desplegar la información que contiene el directorio. Sin embargo, tiene como limitaciones el hecho de que la información disponible es restringida pues no brinda detalles sobre los tipos de problemas jurídicos que son competencia de cada institución, y no se orienta al ciudadano en lo referente a los términos jurídicos y a las competencias asignadas a cada institución.

En síntesis, se evidenció la necesidad de contar con herramientas informáticas, en particular sistemas de información en la web, que permitan administrar la información sobre la oferta de justicia y la forma en que los ciudadanos pueden acceder a cada servicio de justicia disponible en un territorio. Esta necesidad detectada desde el MIJ de Colombia, también ha sido

\footnotetext{
${ }^{2}$ Disponible en www.conciliacion.gov.co

${ }^{3}$ Disponible en www.mjusticia.gob.es

${ }^{4}$ Disponible en www.minjusticia.gob.cl

${ }^{5}$ Disponible en www.jus.gob.ar

${ }^{6}$ Disponible en www.cej.org.py/mapa
} 
abordada en otros países, sin embargo, como se mencionó anteriormente, dichas soluciones presentan algunas debilidades que limitan su impacto en la ciudadanía. En este orden de ideas, en la propuesta de Mapa de Oferta de Justicia que se presenta en este artículo, se busca ofrecer una alternativa de solución a la problemática planteada en donde se recogieron elementos de estos desarrollos similares y se implementaron mejoras para disminuir o eliminar las debilidades detectadas.

\section{METODOLOGÍA}

Para realizar la planificación, ejecución y control del proyecto, el equipo de trabajo seleccionó el método ágil para el desarrollo de software denominado Programación Extrema (Extreme Programming $-X P$ ). Este método ágil fue propuesto como una de las formas de llevar a la práctica los valores definidos en el manifiesto ágil, Beck et al [6]. Su característica principal es la flexibilidad y facilidad de incorporarlo en las prácticas de desarrollo de software en equipos pequeños y medianos, que deban afrontar un proyecto en donde los requisitos tengan una dinámica de cambio rápida, Baird [7]. En este sentido, para el caso del proyecto Mapa de Oferta de Justicia, el principal reto metodológico era que, aunque se tenía establecida una visión global del alcance, los requisitos específicos para cada aplicación no estaban definidos y se esperaba que durante la ejecución del proyecto emergieran todos los detalles para concretarlos. Además, el equipo de trabajo responsable del componente de $\mathrm{TI}$ estaba conformado por ocho personas: dos ingenieros y seis programadores. Es decir, con la estructura de un equipo pequeño. Estos dos hechos motivaron la apropiación y uso del método ágil XP.

En términos generales, el método XP está conformado por cuatro elementos esenciales: valores, principios, actividades y prácticas. Los valores que debe formar un equipo de XP son la simplicidad de las soluciones construidas, la comunicación, priorizando la comunicación oral, como el valor central para que el equipo de trabajo obtenga resultados que cumplan o excedan las expectativas, la realimentación permanente por parte de clientes, y el coraje, entendido como la confianza para trabajar rápido, corregir si es necesario, y tener la certeza de que el código construido supere las pruebas diseñadas para medir su calidad, Beck et al [8].

El ciclo de vida de un proyecto que use el método XP incluye una primera fase de exploración en donde se lanza el proyecto, se establece la visión del alcance con requisitos de muy alto nivel, y se construyen algunos prototipos exploratorios para determinar aspectos relacionados con la tecnología por usar en el desarrollo. Por otra parte, la planificación y el refinamiento del plan, es una fase que se ejecuta en múltiples ocasiones, allí se determinan las prioridades en las funcionalidades por construir, y se establece la estimación de tiempos para realizar entregas parciales del producto. El proceso iterativo de construcción también involucra actividades como la comunicación con el cliente para definir "historias de usuario", la especificación de las pruebas que debe pasar el código para satisfacer las funcionalidades especificadas en las "historias de usuario" y, el diseño, la construcción y la prueba de código 
fuente para implementar las funcionalidades requeridas. En la Figura 1 se presenta un diagrama con el ciclo de vida de un proyecto que use como guía el método XP.

Cómo se ve en la Figura 1, después de la exploración, el equipo de trabajo se involucra en un proceso iterativo de planificación y construcción de versiones incrementales del producto, también denominadas liberaciones (reléase), que tan pronto se construyen pasan a ser utilizadas por los usuarios, con el propósito de tener realimentación sobre la funcionalidad liberada y poder realizar mantenimiento, así como, tener en cuenta esas observaciones en la ejecución de las siguientes iteraciones. Estos aspectos relacionados con el enfoque iterativo e incremental, y la posibilidad de poner en producción liberaciones tempranas de los productos, son importantes para proyectos como el Mapa de Oferta de Justicia, en donde, además de construir el producto, era necesario avanzar simultáneamente en la recopilación de los datos que se procesarían con el producto.

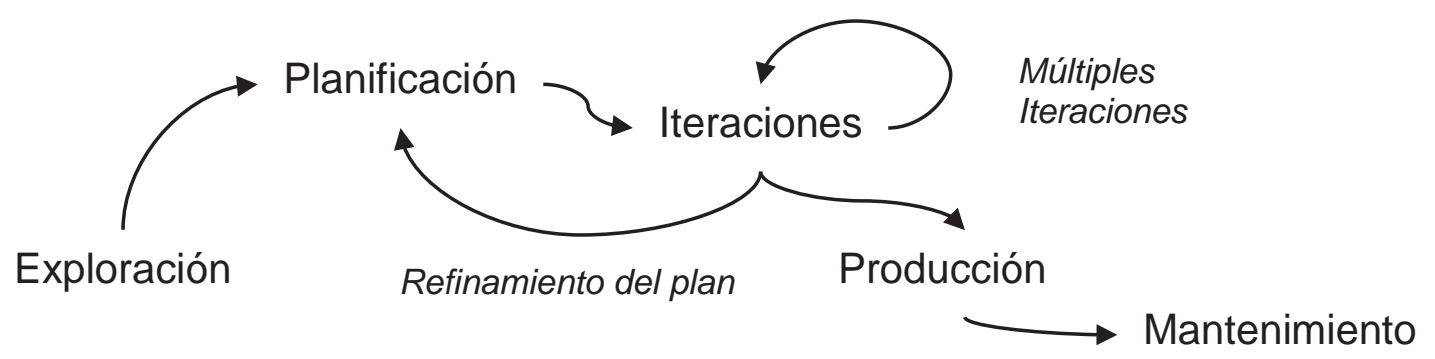

Figura 1. Ciclo de vida de un proyecto basado en XP

Fuente: Adaptada de Baird, 2002

La ejecución del proyecto con base en el ciclo de vida descrito se inició con la exploración. Aquí se definió por parte del equipo de desarrollo y los representantes del MIJ, que la solución propuesta para apoyar los procesos de administración de información del sistema de justicia colombiano, sería integrar tres aplicaciones software en ambiente web, denominadas Tesauro, Directorio de Instituciones Oferentes de Servicios de Justicia y Plataforma de consultas y reporte de indicadores, Vera et al [3]. Esta conceptualización se sintetizó en el diagrama que se presenta en la Figura 2, en donde se muestran las aplicaciones desarrolladas y la interacción con diferentes tipos de usuario.

Sumado a esto, se establecieron restricciones técnicas para el desarrollo. En este sentido, es importante destacar que se definió que las aplicaciones ofrecieran su funcionalidad con una interfaz de usuario Web, con la cual se puede interactuar a través de un browser para navegar en Internet. Además, se estableció que el desarrollo de las aplicaciones se hiciera utilizando la versión 2.0 del Framework. Net de Microsoft, específicamente la tecnología ASP.NET 2.0 y el lenguaje de programación C\#, incluyendo componentes de AJAX para enriquecer la interacción con el usuario. Como guía para el desarrollo basado en ASP.NET se utilizaron como referencia los trabajos de Macdonaldy et al [9], Evjen et al [10] y Murach et al [11]. 


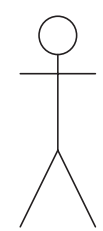

Usuario de Servicios de Justicia

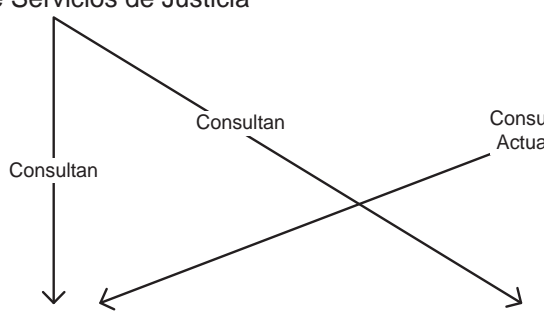

\section{Aplicación Web 1}

Directorio de Instituciones Oferentes de Servicios de Justicia en Colombia
Aplicación Web 3

Tesauro sobre el

Funcionamiento de la Oferta de Justicia en Colombia

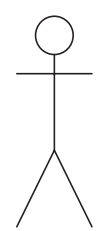

Instituciones Oferentes de Servicios de Justicia
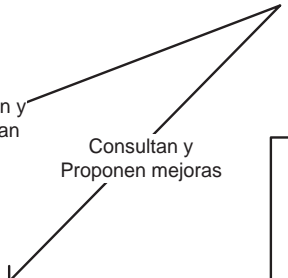

Fuentes de datos externas (por ejemplo datos demográficos, socio políticos, económicos)

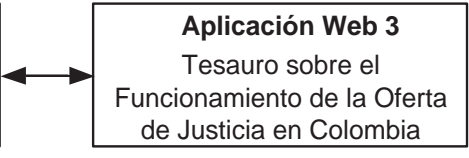

Aplicación Web 2

Plataforma de Consultas y Reportes de Indicadores sobre Oferta de Justicia en Colombia
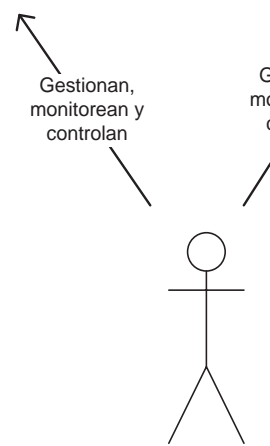

Ministerio del Interior y de Justicia
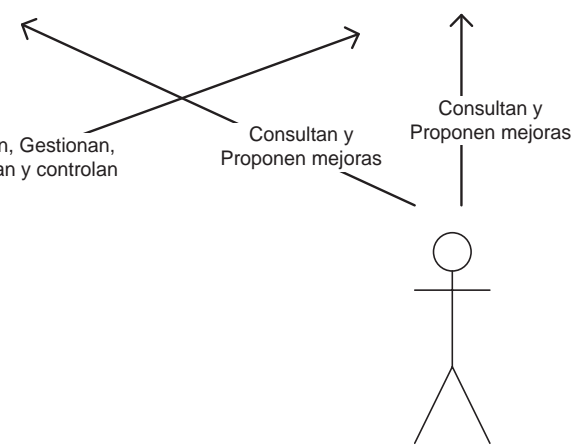

Investigadores Socio Jurídicos

Figura 2. Visión general de la plataforma de TI desarrollada Fuente: Adaptada de Vera et al 2008.

Así mismo, la persistencia de los datos se concibió con el soporte de tres bases de datos relacionales implementadas en el motor de bases de datos Microsoft SOL Server 2005, para lo cual se utilizó como guía lo propuesto en Rankins et al [12]. Adicionalmente, para la aplicación de Consultas y Reportes de Indicadores se determinó la necesidad de utilizar los servicios de integración (SOL Server Integration Services) y de reportes (SOL Server Reporting Services) que ofrece el producto Microsoft SOL Server 2005, tal y como se presentan en los trabajos de Larson [13], Knight et al [14], Turley et al [15], y Tang et al [16]. Por último, para las características de usabilidad se estableció que debían seguir las mejores prácticas propuestas por el departamento de salud del Gobierno de Estados Unidos, disponibles en $\mathrm{O}$ Leavitt et al [17]. Además, cabe destacar que para el desarrollo de aplicaciones se mantuvo comunicación 
directa con el equipo de ingenieros del Ministerio del Interior y Justicia, quienes entregaron los estándares para el diseño gráfico y las indicaciones pertinentes para el proceso de implementación de las aplicaciones web en su infraestructura.

A partir del resultado de la fase de exploración se tomó la decisión de dividir el proyecto en tres sub-proyectos, uno para cada aplicación, de modo que las fases subsiguientes se planificaran y ejecutaran en forma paralela para las tres aplicaciones. Para la planificación inicial se construyeron diagramas de casos de uso para representar gráficamente los requisitos funcionales de cada aplicación, y se describió un conjunto de historias de usuario, que es el artefacto propuesto por la metodología XP para recoger la descripción de la funcionalidad necesaria, así como, elementos de la estimación, priorización y asignación de la implementación en el equipo de desarrolladores. En este sentido, para la aplicación Tesauro, se modelaron cuatro actores y ocho casos de uso, como se ve en la Figura 3.

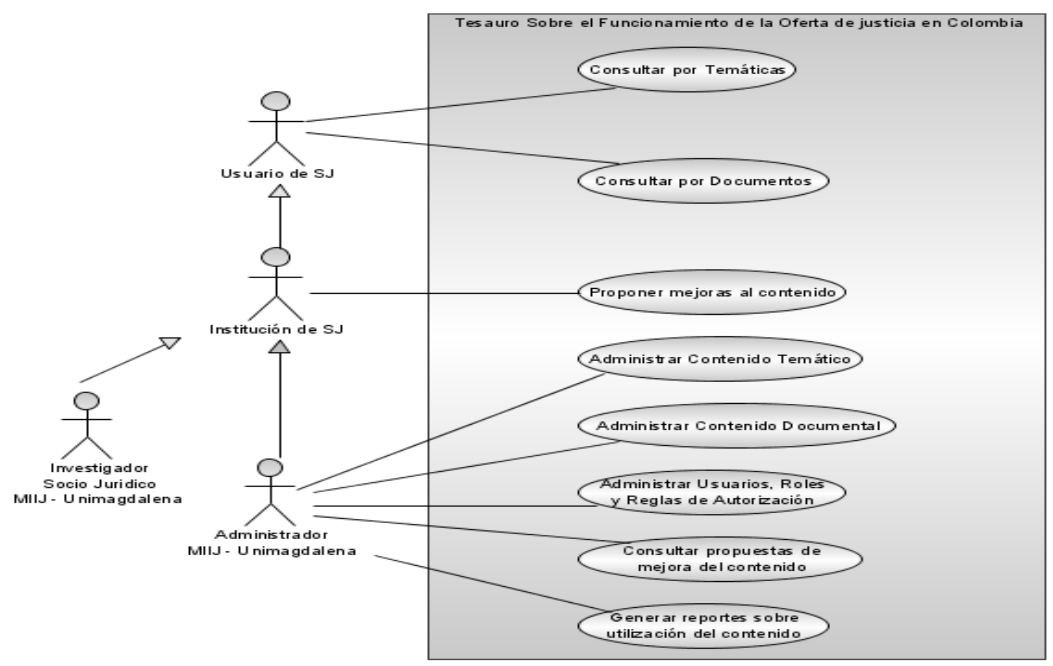

Figura 3. Diagrama de casos de uso de la Aplicación "Tesauro" Fuente: Adaptada de Vera et al, 2008 y Vásquez et al, 2008.

De la misma forma, para aplicar el Directorio se modelaron cuatro actores y 22 casos de uso, como se ve en la Figura 4. Así mismo, para aplicar la Plataforma de Reportes se modelaron dos actores y 12 casos de uso, lo cual se muestra en la Figura 5.

A partir del modelado de la funcionalidad con los casos de uso, se procedió a construir una descripción más detallada de la funcionalidad necesaria utilizando el artefacto denominado historia de usuario. Una vez definidas las historias, el equipo realizó la estimación del tiempo necesario para implementar cada una y después se procedió a realizar la priorización con el cliente. De esta manera se facilitó trazar el plan de lanzamiento en función de dos parámetros: tiempo y grado de importancia para el cliente. 
142

TECNOLOGÍAS DE INFORMACIÓN PARA ACERCAR AL CIUDADANO A LOS SERVICIOS DE JUSTICIA EN COLOMBIA:

EL CASO DEL MAPA DE OFERTA DE JUSTICIA

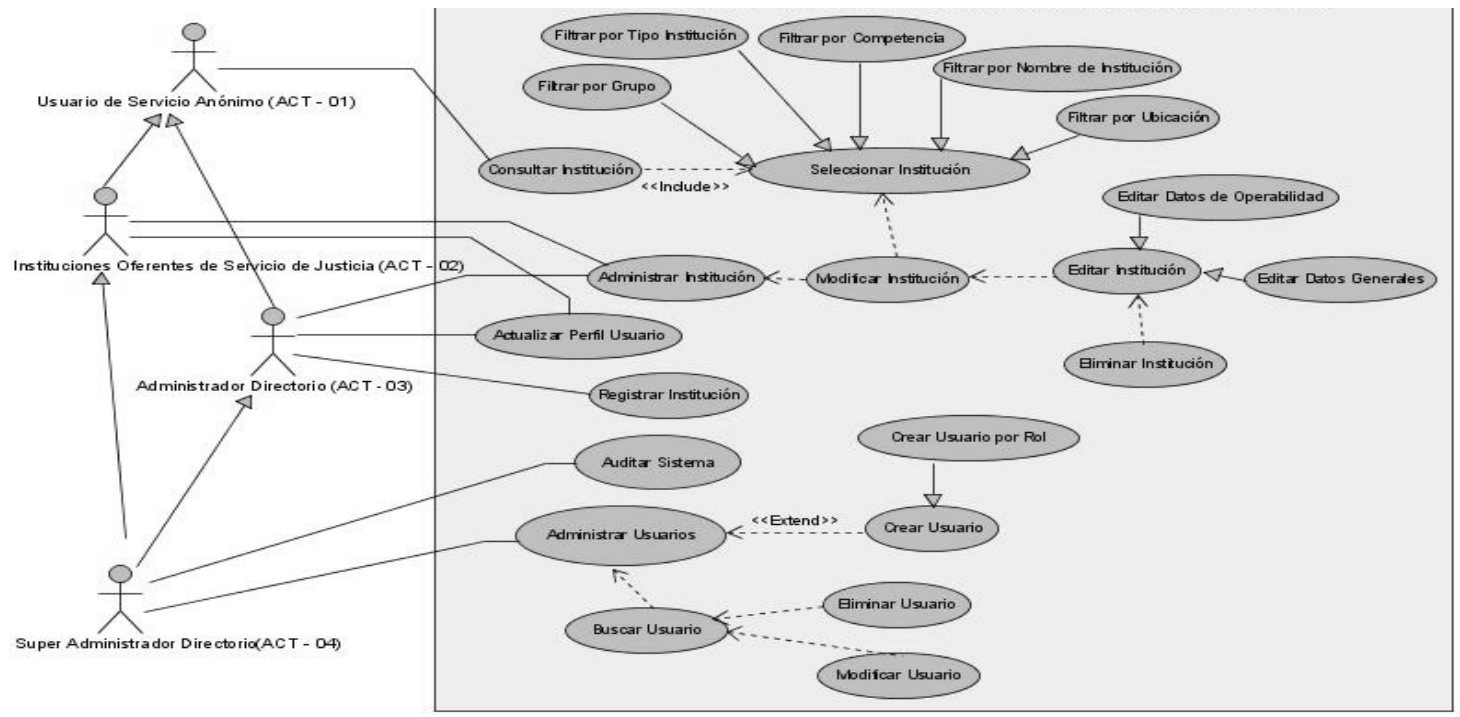

Figura 4. Diagrama de casos de usos de la aplicación "Directorio"

Fuente: Adaptada de Vera et al, 2008 y Arias et al, 2008.

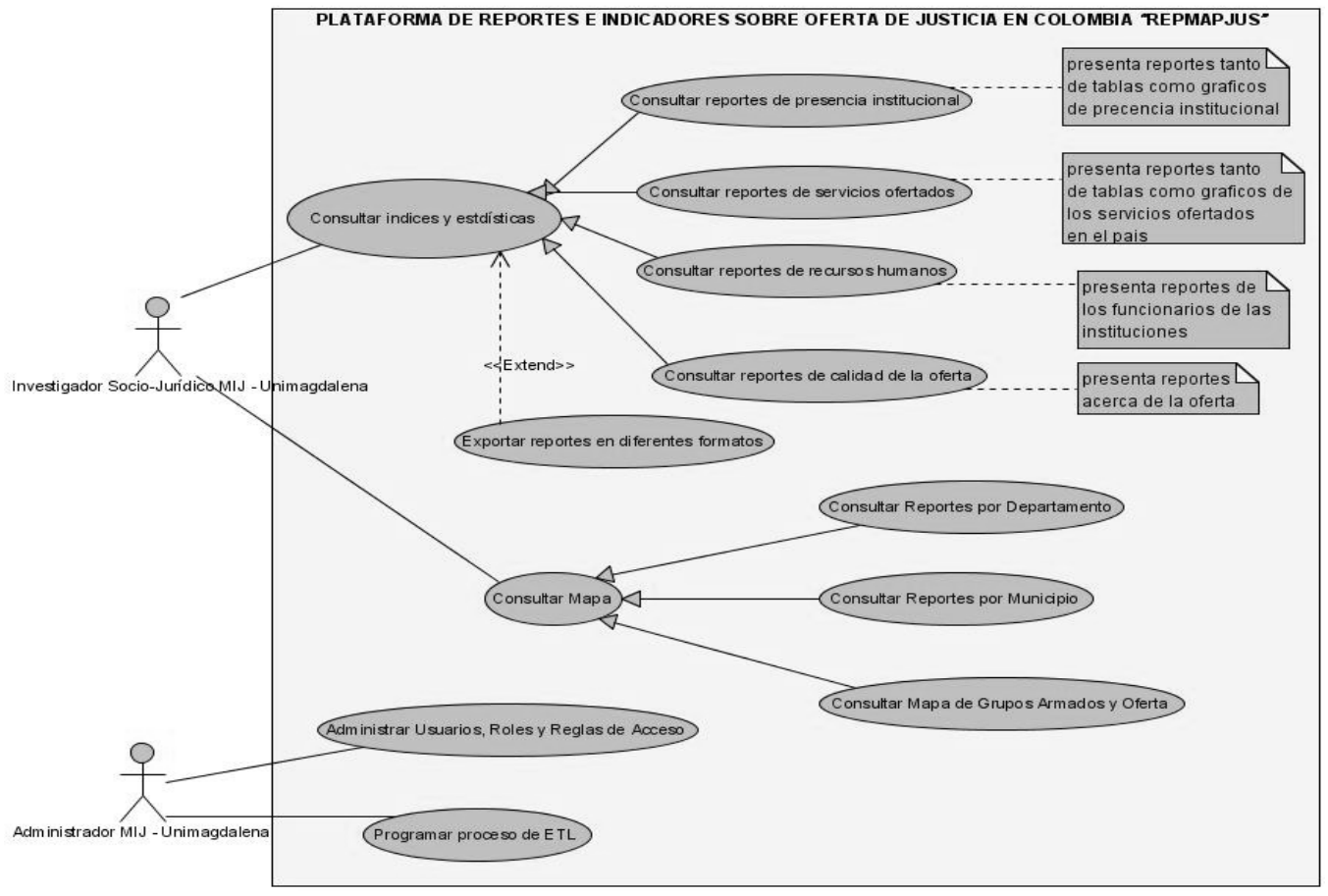

Figura 5. Casos de uso de Aplicación "Plataforma de Consultas y Reportes de Indicadores"

Fuente: Adaptada de Vera et al, 2008 y Martes et al 2008

ERNESTO AMARU GALVIS LISTA, MAYDA PATRICIA GONZÁLEZ ZABALA, PABLO HERNÁNVERA SALAZAR 
Tabla 3. Ejemplo de una historia de usuario de la aplicación "Directorio"

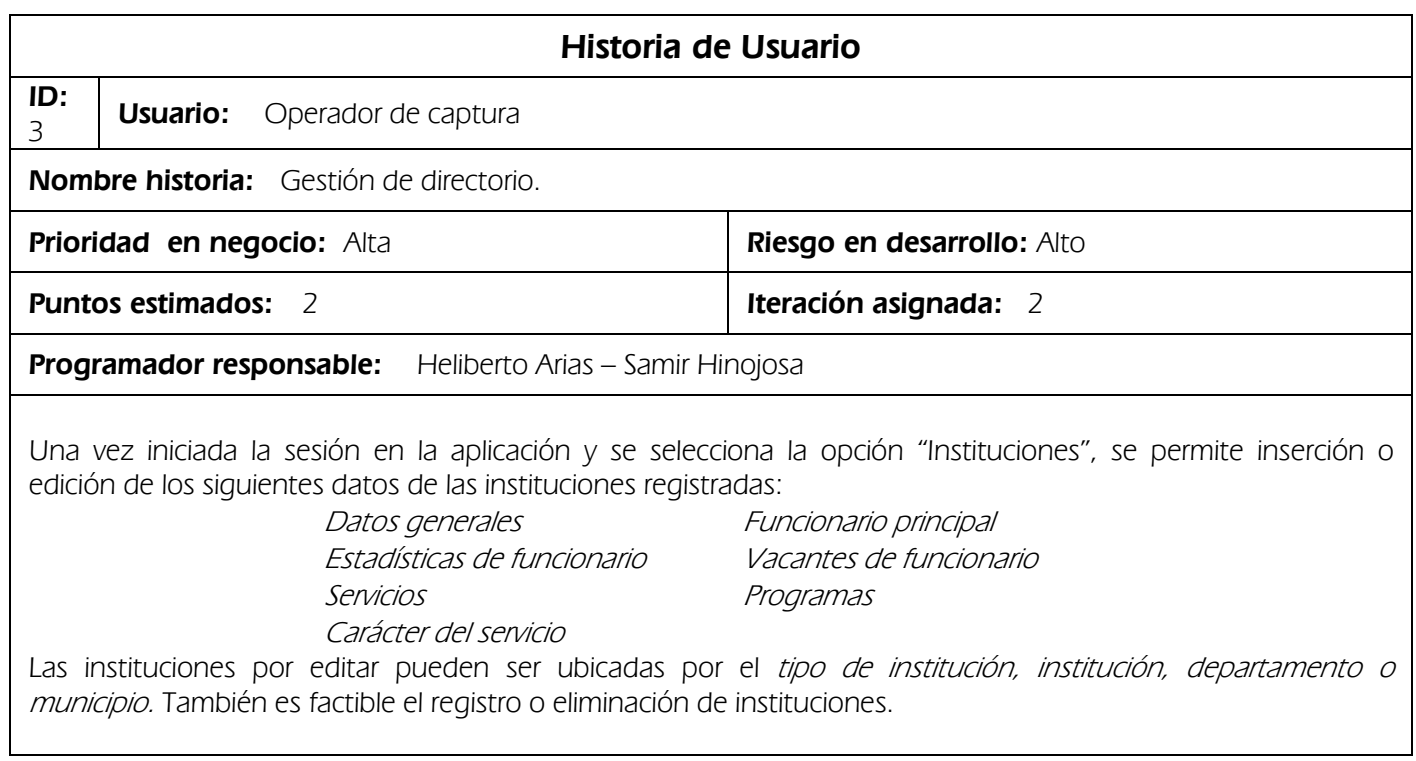

Fuente: Tomada de Arias et al, 2008.

En la Tabla 3 se presenta un ejemplo de una historia de usuario de la aplicación Directorio de Oferta de Justicia, y en la Tabla 4 se presenta el resumen de la cantidad de historias de usuario construidas para cada aplicación, y el número de iteraciones planificadas para su desarrollo.

Tabla 4. Resumen del resultado de la planificación en términos de historias de usuarios e iteraciones

\begin{tabular}{|l|c|c|}
\hline Aplicación & Historias de usuario & Iteraciones \\
\hline Directorio & 13 & 6 \\
\hline Tesauro & 8 & 6 \\
\hline Reportes & 12 & 6 \\
\hline
\end{tabular}

Después se ejecutó cada una de las iteraciones siguiendo el proceso establecido en la metodología, en el cual se diseñaban las pruebas para las historias de usuario cubiertas en la iteración, y se procedía a diseñar, construir y probar la funcionalidad necesaria. Al finalizar la ejecución de cada iteración se hace una liberación de la funcionalidad implementada para que el equipo jurídico del proyecto procediera a utilizarla en la captura y el procesamiento de los datos. Por otra parte, el equipo de desarrollo también realizaba actividades de revisión de la planificación inicial y de soporte de la versión liberada como resultado de la iteración. Cuando todas las historias de usuarios se implementaron, se dio por terminado el desarrollo de las aplicaciones y se procedió a realizar su entrega y despliegue en la infraestructura del MIJ, incluída la documentación técnica y de usuario. 


\section{RESULTADOS OBTENIDOS}

\subsection{APLICACIÓN DE LA ADMINISTRACIÓN DE UN TESAURO DE TÉRMINO JURÍDICOS}

La aplicación de administración del tesauro permite al público en general consultar situaciones jurídicas comunes, brindando una explicación acerca de cada problemática y presentando una guía de acción en relación con cada una de estas, específicamente a dónde acudir, qué hacer, cuánto puede valer el servicio jurídico, si es necesario o no recurrir a un abogado e información que complementan las descripciones realizadas. Las temáticas se presentan mediante:

- $\quad$ Listado sistemático: en esta forma, se presenta el listado de las temáticas que se han documentado en la aplicación.

- $\quad$ Listado alfabético: se presentan las temáticas en orden alfabético.

- Búsquedas por términos, los cuales están relacionados con las problemáticas.

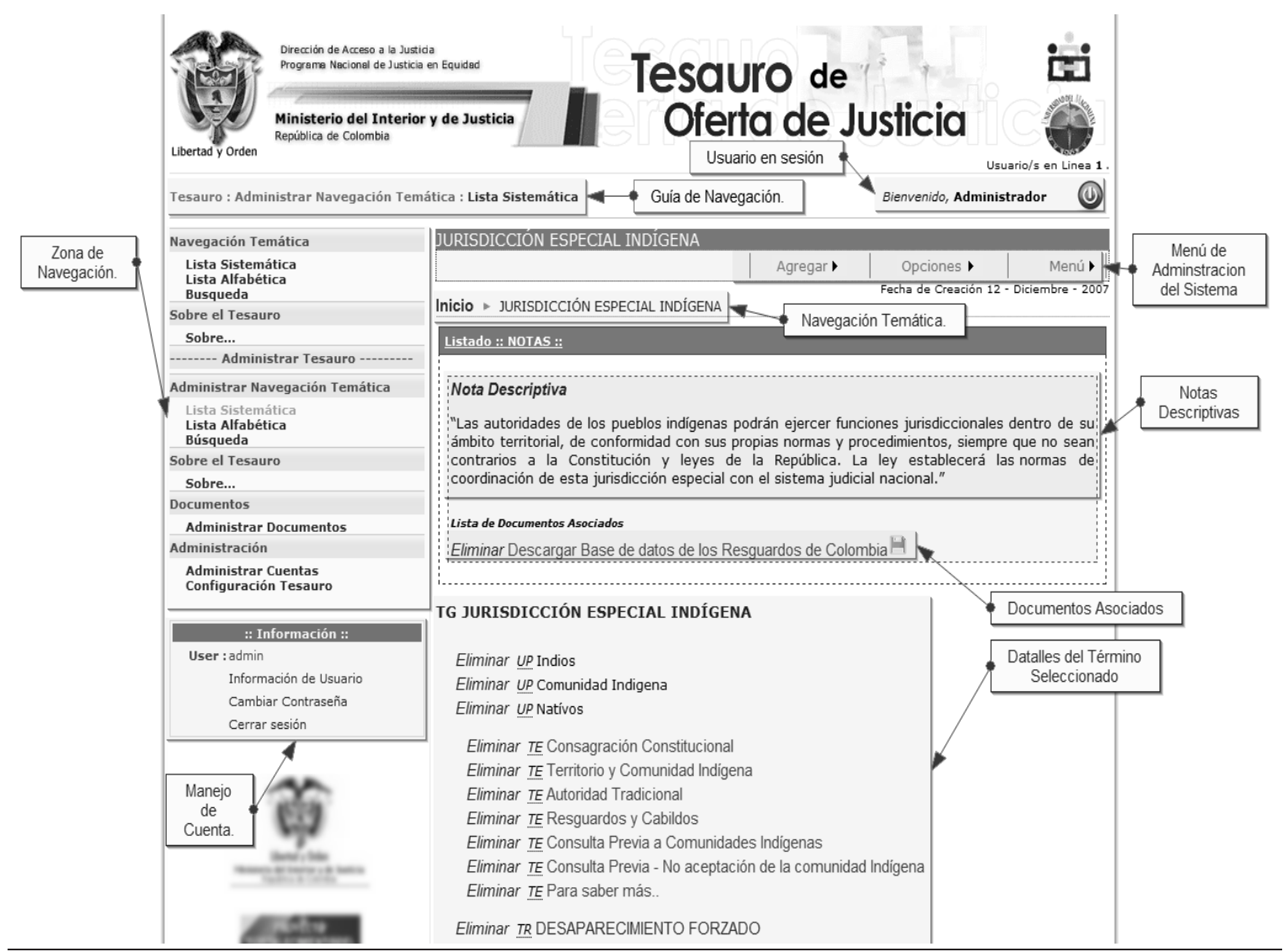

Figura 6. Ejemplo de interfaz de la Aplicación “Tesauro"

Fuente: Tomada de Vásquez et al, 2008. 
Por otra parte, la aplicación permite administrar el contenido, es decir, los términos preferidos, los descriptores, las relaciones jerárquicas entre los temas, las definiciones de los términos y las reglas de acceso a los contenidos. Sumado a esto, la aplicación permite administrar usuarios, roles y reglas de autorización, Vásquez et al [18].

La alimentación inicial del tesauro fue hecha por los investigadores socio jurídicos, específicamente los profesionales del derecho que participan en el proyecto. Luego, se espera que el tesauro ofrezca la funcionalidad de registrar observaciones sobre el contenido, de modo que pueda actualizar y mejorarse con el tiempo. En la Figura 6 se presenta un ejemplo de interfaz de usuario.

\subsection{APLICACIÓN DIRECTORIO DE INSTITUCIONES OFERENTES DE SERVICIOS DE JUSTICIA EN COLOMBIA}

La aplicación del directorio de instituciones permite recopilar y administrar los datos básicos de cada institución que ofrece servicios de justicia en Colombia. Estos datos están relacionados directamente con la presencia institucional, las competencias y los servicios ofrecidos por cada institución. Además, permite registrar datos de los funcionarios responsables de la institución, su tipo de vinculación y las vacantes que existen.

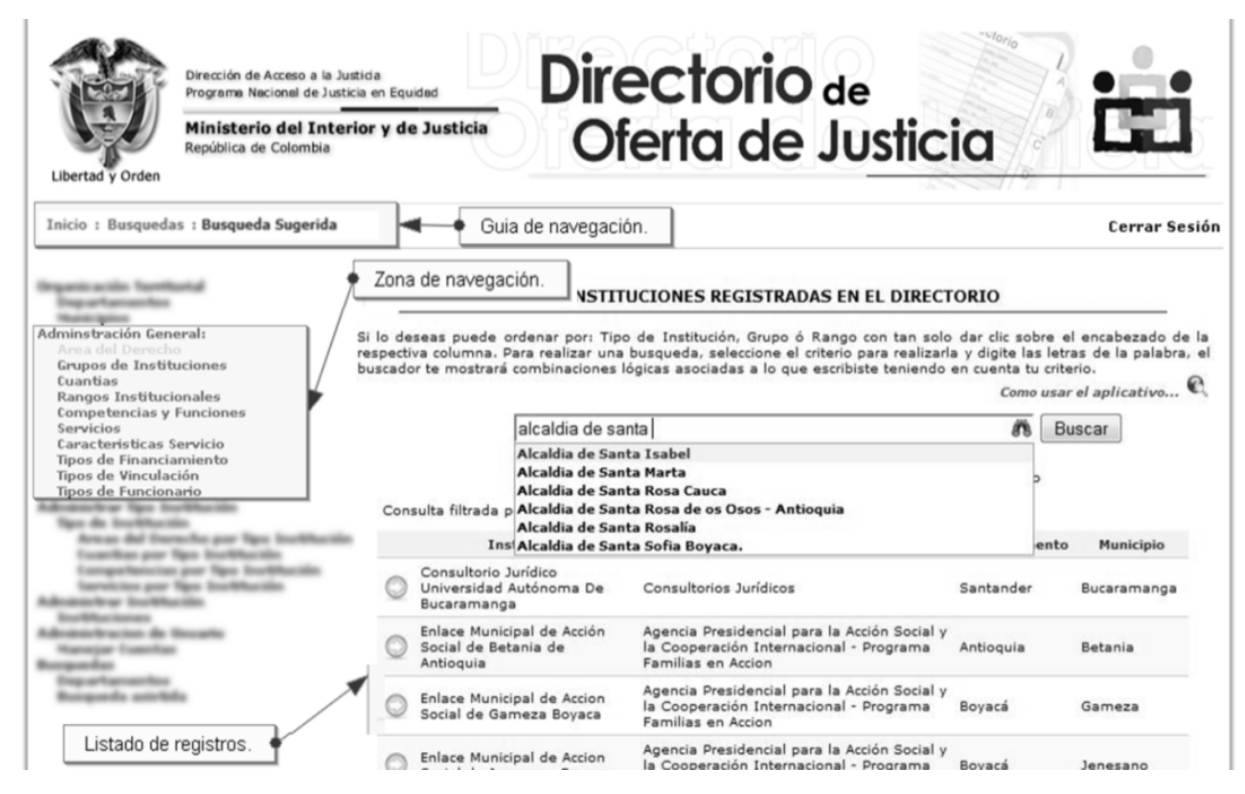

Figura 7. Ejemplo de interfaz de la aplicación "Directorio"

Fuente: Tomada de Arias et al, 2008

Para alimentar inicialmente la base de datos se utilizaron datos recopilados directamente la través de llamadas telefónicas y correo electrónico) y en fuentes secundarias (documentos institucionales y estudios disponibles para consultal. No obstante, el software permite la 
actualización constante, que debe ser coordinada por el MIJ y las instituciones interesadas, Arias et al [19]. Está aplicación permite administrar:

- Instituciones

- $\quad$ Tipos de instituciones: asignar y/o modificar las características generales de cada tipo de institución, específicamente en cuanto: Áreas del derecho que trabaja el tipo de institución, cuantías, competencias y servicios.

- Organización territorial, es decir crear o modificar zonas territoriales como los departamentos y sus municipios.

- $\quad$ Datos de referencia, para especificar y modificar los criterios al registrar la información. Para el caso de la aplicación se definieron como términos de referencia: áreas del derecho, grupos de instituciones, cuantías, rango (nacional o local), competencias, servicios, tipos de financiamiento, y tipos de funcionarios.

- $\quad$ Cuentas de usuarios y sus registros, así como controles de acceso.

- $\quad$ Proceso de auditoría sobre el registro y modificación de información de las instituciones, con lo cual se tiene control en cambios de la información.

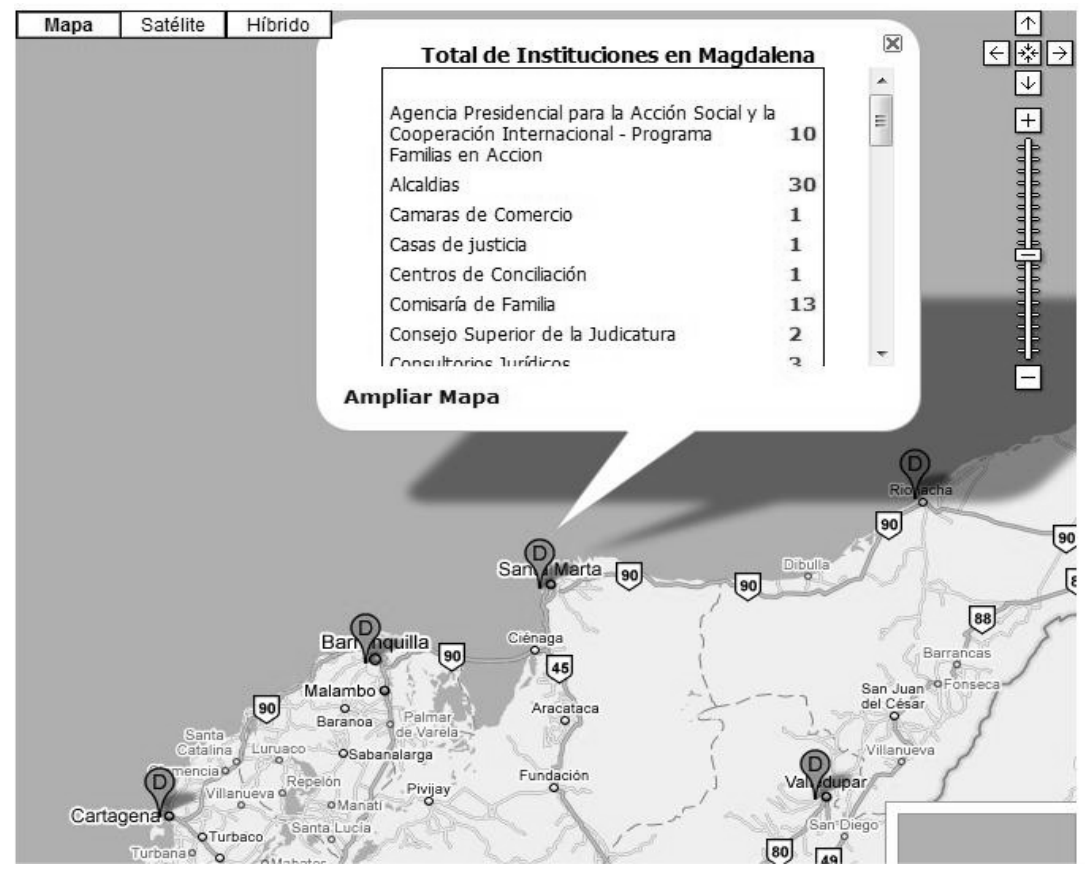

Figura 8. Ejemplo de consulta del "Directorio" utilizando la tecnología de Google Maps.

Por otra parte, la aplicación permite adelantar búsquedas por ubicación, tipo de competencias y funciones, además de generar informes. Finalmente, es importante aclarar que la aplicación se entrega con la alimentación realizada por los investigadores socio jurídicos involucrados en el proyecto, Arias et al [19]. En la Figura 7 se puede apreciar una interfaz de la aplicación en 
donde se listan todas las instituciones registradas en el directorio y se puede realizar búsquedas.

Además, en esta aplicación se implementó la presentación de información con el servicio de Google Maps, en el cual, al seleccionar la zona geográfica que se desea consultar, se podrá visualizar información sobre las instituciones que existen en ese territorio. En la Figura 8 se presenta un ejemplo de consulta sobre las instituciones registradas en el departamento del Magdalena.

\subsection{APLICACIÓN PLATAFORMA DE CONSULTAS Y REPORTES DE INDICADORES}

La aplicación denominada Plataforma de consultas y reportes de indicadores, permite consultar los indicadores de oferta de justicia y genera reportes específicos de forma gráfica y en tablas, necesarios para llevar a cabo análisis sobre el área de interés (oferta de justicia) y la posterior toma de decisiones estratégicas y tácticas, Martes et al [20].

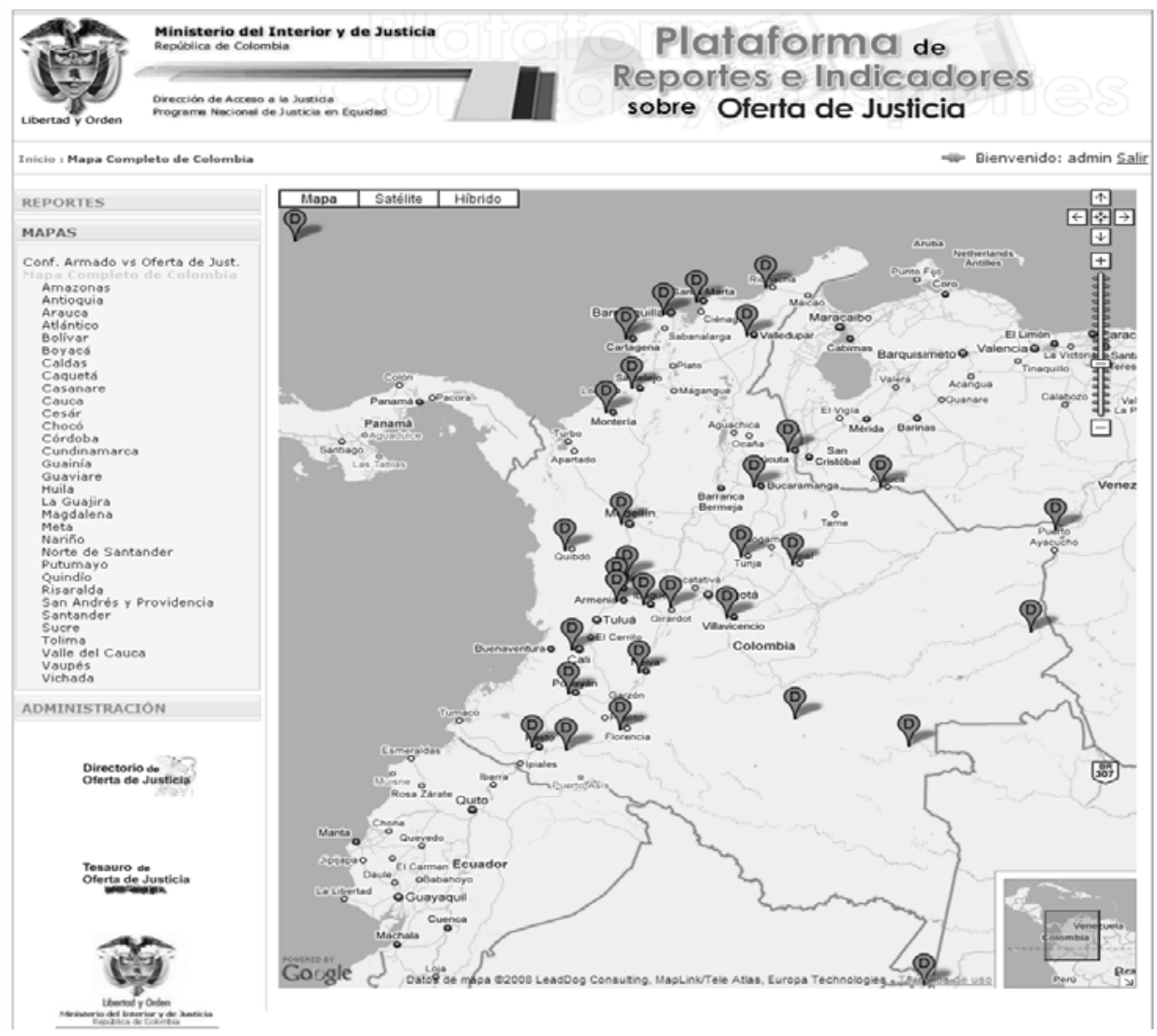

Figura 9. Ejemplo de interfaz de la Aplicación Plataforma de Consultas y Reportes de Indicadores

Fuente: Tomada de Martes et al, 2008 
El insumo primario para esta plataforma son los datos disponibles en el "Directorio de Instituciones Oferentes de Servicios de Justicia en Colombia", sobre los cuales se realiza un proceso de extracción, transformación y carga, en una estructura de almacenamiento diseñada para facilitar la generación de consultas, reportes e indicadores. Por esta razón los reportes que se generan en esta aplicación reflejan la información recopilada en el directorio. Adicionalmente, esta plataforma integra, de forma básica, datos geográficos y demográficos, necesarios para realizar los cálculos estadísticos que requieren los investigadores socio jurídicos. En este sentido, la aplicación implementa el sistema de muestra de indicadores a través del servicio web Google Maps, en donde al seleccionar la zona geográfica que se desea consultar, se podrá visualizar información y datos estadísticos sobre ella. De igual manera, la aplicación permite administrar los usuarios, los controles de acceso y la determinación de los períodos de carga de datos (rango de tiempo que se estipulan para el análisis de información). Finalmente, se especifica que la aplicación se entrega con la alimentación realizada con la aplicación del Directorio de Instituciones Oferentes de Servicios de Justicia en Colombia, es decir toma los datos introducidos por los investigadores socio jurídicos involucrados en el proyecto y los analiza de acuerdo con los criterios establecidos. En la Figura 9 se presenta un ejemplo de la interfaz.

\section{CONCLUSIONES}

La ejecución de las aplicaciones que conforman la plataforma de TI propuesta se realizó gracias a un trabajo interdisciplinario, que permitió desarrollar una solución informática que fuera pertinente a la problemática detectada. Así mismo, el uso de la metodología ágil XP, facilitó la interacción con el equipo jurídico del proyecto, permitiendo definir los requisitos funcionales y no funcionales para cada producto elaborado.

En cuanto a las aplicaciones, el desarrollo del Directorio de instituciones oferentes de justicia permitió recopilar y unificar la información disponible y permitió determinar la presencia institucional y los servicios disponibles en el País. En relación con la aplicación de consultas y reportes, se logró obtener información que sintetiza aspectos relevantes sobre el estado de la oferta de justicia en el País en un momento dado, a manera de indicadores de diferentes tipos, para apoyar procesos de estudio y toma de decisiones en diferentes niveles (estratégico, táctico y operativo). Cabe destacar que el insumo para lograr dichos indicadores proviene de aplicar el directorio de instituciones. A su vez, la aplicación Tesauro puede convertirse en un medio que facilite el acercamiento y entendimiento de los ciudadanos respecto de los servicios de justicia y a la vez ofrece una guía sobre el qué, cómo hacer y el dónde acudir.

Por otra parte, la exitosa ejecución del proyecto le permitió a la Universidad del Magdalena fortalecer sus vínculos de cooperación con el Misterio del Interior y Justicia, así como con otras instancias del Gobierno nacional. Esto posibilitó el desarrollo posterior de convenios de cooperación para ejecutar proyectos en todo el territorio nacional. En este mismo sentido, se llevó a cabo una actividad de socialización con líderes sociales y conciliadores en equidad para manejar la aplicación, que en el futuro, de la mano de la estrategia de masificación del acceso 
a Internet, podría convertirse en una alternativa para mejorar el acceso de los ciudadanos a la oferta de justicia, en particular en el marco de la Ley de Víctimas y de Restitución de Tierras que tendrá una alta demanda en la región Caribe.

En relación con las implicaciones internas de la ejecución del proyecto, se evidenció que la participación de docentes y estudiantes del Programa de Ingeniería de Sistemas en el proyecto liderado por la Vicerrectoría de Extensión, permitió la articulación entre las funciones de docencia, investigación y extensión. Así mismo, permitió desarrollar productos orientados a solucionar problemas del entorno.

Además se muestra cómo las alianzas de cooperación entre entidades del Gobierno y las universidades pueden contribuir a mejorar la prestación de servicios al ciudadano, sin que se desvirtúe la naturaleza académica de la universidad. Por el contrario, se convierte en una oportunidad para desarrollar investigación aplicada y pertinente a las necesidades del País.

Por último, es importante destacar que el alcance logrado hasta el momento debe complementarse con varios esfuerzos futuros como: (1) programas de divulgación y capacitación por parte del ente encargado de administrar este sistema, de modo que se generalice su uso, al menos en las instituciones que siven de guía a los ciudadanos para acceder a la justicia; (2) actualización de los datos registrados en la base de datos del directorio de instituciones, en particular la referente a los funcionarios encargados de brindar los servicios en cada institución, pues con los procesos propios de la democracia, en cada cambio de administración local, regional o nacional, se generan cambios en las personas responsables de las instituciones; (3) ampliación de la información disponible en la aplicación del tesauro a partir de la realimentación obtenida de los usuarios y de estudios socio-jurídicos en diversas regiones del País; (4) ampliación de las funcionalidades de búsqueda en las tres aplicaciones; (5) integración de aplicaciones web, con otros servicios de información del Estado; (6) construcción de versiones de las aplicaciones Tesauro y Directorio para dispositivos móviles; y (7) evaluación del impacto que tiene el uso de estas herramientas en el acceso a la justicia por parte de los ciudadanos colombianos.

\section{AGRADECIMIENTOS}

Los autores expresan sus agradecimientos al MIJ y a la Vicerrectoría de Extensión de la Universidad del Magdalena por su apoyo en la ejecución del presente proyecto.

\section{REFERENCIAS BIBLIOGRÁFICAS}

[1] Vicerrectoría de Extensión (2007), Estudios previos del convenio Ministerio del Interior y Justicia - Universidad del Magdalena, Informe técnico Universidad del Magdalena, Santa Marta. 
[2] Vicerrectoría de Extensión, (2007). Términos de Referencia convenio Ministerio del Interior y Justicia - Universidad del Magdalena. Informe técnico Universidad del Magdalena, Santa Marta.

[3] Vera P., Díaz M., Galvis E.A. y González Zabala M. P., (2008). Informe Final - Proyecto Mapa de Oferta de Justicia en Colombia y Diplomados para la Formación de Gestores Municipales del Programa Nacional de Justicia en Equidad. Universidad del Magdalena, Santa Marta. Informe técnico.

[4] DANE, (2007) Sistema de Consulta de la División Político - Administrativa de Colombia, Sistema de información geoestadística. En: http://190.25.231.236/Divipola/Divipola.asp.

[5] Pérez Luño A.E. Garzón Valdés D. L. S., y Vázquez D. L. S., (1996). Ensayos de informática jurídica.

[6] Beck K., Beedle M., Van Bennekum A., Cockburn A., Cunningham W., Fowler M., Grenning J., Highsmith J., Hunt A., Jeffries R., Kern J., Marick B., Martin R. C., Mellor S., Schwaber K., Sutherland J., and Thomas D., (2001). Manifesto for Agile Software Development.

[7] Baird S., (2002). Sams Teach Yourself Extreme Programming in 24 Hours, Sams.

[8] Beck K., and Andres C., (2004). Extreme Programming Explained: Embrace Change, 2. ed. Addison-Wesley Professional.

[9] MacDonald M. y Szpuszta M., (2005). Pro ASP.NET 2.0 in C\# 2005, A-Press.

[10] Evjen B., Hanselman S., Muhammad F., Sivakumar S. and Rader D., (2005). Professional ASP.NET 2.0, 4. ${ }^{\mathrm{a}}$ ed. Wrox.

[11] Murach J., and Boehm A., (2006). Murach's ASP.NET 2.0 Web Programming with C\# 2005. Mike Murach \& Associates.

[12] Rankins R., Bertucci P., Gallelli C., and Silverstein A.T. (2006). Microsoft SOL Server 2005 Unleashed. Sams.

[13] Larson B., (2006). Delivering Business Intelligence with Microsoft SOL Server 2005: Utilize Microsoft's Data Warehousing, Mining \& Reporting Tools to Provide Critical Intelligence to A. McGraw-Hill Osborne Media.

[14] Knight B., Mitchell A., Green D., Hinson D., Kellenberger K., Leonard A., Veerman E., Gerard J.H., Ji, and M. Murphy, (2006). Professional SOL Server 2005 Integration Services, 1. ${ }^{\text {a }}$ ed. Wrox. 
[15] Turley P., Bryant T., Counihan J., and DuVarney D., (2006). Professional SOL Server 2005 Reporting Services, 2nd ed. Wrox, 2006.

[16] Tang Z. and MacLennan J., (2005). Data Mining with SOL Server 2005, 1. a ed. Wiley.

[17] Leavitt M.O., and Shneiderman B., (2006). Research-based web design \& usability guidelines. Washington: U.S. Department of Health and Human Services.

[18] Vásquez R., y Caro R., (2008). Sistema de información para la administración de un tesauro sobre problemas jurídicos y la oferta de servicios de justicia en Colombia TESMAPJUS, Trabajo de Grado, Universidad del Magdalena, Santa Marta - Colombia.

[19] Arias H., y S. Hinojosa, (2008). Sistema de información para administrar el directorio de instituciones oferentes de servicios de justicia en Colombia - DIRMAPJUS, Trabajo de Grado, Universidad del Magdalena, Santa Marta - Colombia.

[20] Martes P. y Velázquez E., (2008). Plataforma de reportes e indicadores sobre oferta de justicia en Colombia - REPMAPJUS, Trabajo de Grado, Universidad del Magdalena, Santa Marta - Colombia. 
